\title{
Pathogenic potential of Escherichia coli from polymicrobial urinary tract infections
}

In a recent issue of this journal, Croxall et al. (2011) described in their work on polymicrobial urinary tract infections (UTIs) data about the pathogenic potential of Escherichia coli isolates. I have several comments on their study regarding the method used by the authors for the research that I think has invalidated the results, which are in discord with the literature. I also believe that the authors omitted consideration of some previous studies that described basic findings on the connection between E. coli pathogenicity and the susceptibility to the antibiotic quinolone. In addition, I think that a more critical view should have been taken in the interpretation of the results.

Croxall et al. (2011) showed an increased human pathogenic potential of E. coli strains isolated from polymicrobial urine, with respect to those from monomicrobial urine, among elderly patients. Although this field is undervalued by the scientific community, from a diagnostic and therapeutic point of view it is of high interest and importance. Therefore, I believe the results reported by Croxall et al. (2011) warrant several comments about the method chosen by the authors.

Firstly, urine samples were assigned to five different categories, where polymicrobial samples were subdivided into male, female, non-catheterized and catheterized samples, and monomicrobial samples were not divided. These categories were used for the analysis of the prevalence of each of the main bacterial species responsible for UTIs, and the relative results showed data in accordance to the literature (Foxman \& Brown, 2003; Ronald, 2003), both in the comparison between genders, noncatheterized and catheterized subjects, and polymicrobial and monomicrobial infections, supporting the choice of method. However, the susceptibility to antibiotic drugs was instead presumably evaluated (since results are not shown) regardless of the aforementioned categories of patients. Also, the authors considered all micro-organisms and all drugs together. Therefore, the analysis led to comparable levels of antibiotic resistance between mixed and monomicrobial culture samples. This finding is in discord with the literature, in which it is well documented that mixed urinary infections are the most refractory to most front-line antibiotics. This phenomenon is acknowledged to be due to the great prevalence of mixed infections in compromised patients, particularly in those who were catheterized (Houdouin et al., 2007), among whom the highest prevalence of resistance towards antibiotics does exist (Domann et al., 2003; Siegman-Igra et al., 1994).

Secondly, the same analysis led to a prevalence of ciprofloxacin-resistant E. coli strains in polymicrobial samples, with respect to monomicrobial samples. This finding, although not discordant with data in the literature, is in discord with the results of a recent study by myself and my colleagues (Saverino et al., 2011), and contrasts with the other important point emerging from the work by Croxall and colleagues, which is the increased pathogenic potential of E. coli strains derived from polymicrobial UTIs. In their work on bacterial pathogenicity, the authors listed isolate resistance to a wide range of antibiotics and they did not refer to the fundamental relationship between the few virulence factors of certain E. coli strains, and their quinolone and fluoroquinolone resistance, nor to the still topical debate concerning underlying mechanisms (Platell et al., 2010; Takahashi et al., 2009), started by Johnson in 2005 (Johnson, 2005). In the opinion of myself and my collaborators, this basic biological correlation and medical finding cannot be left out of consideration any more. Already in a basic review about virulence factors in E. coli cited by the authors (Johnson, 1991), Johnson doubted that the currently defined virulence factors, at that time, could be sufficient in themselves to transform an avirulent organism into a pathogen, demonstrating that other as-yetundefined virulence properties awaited discovery. Following the findings made in previous studies, some of which were referenced by Croxall and colleagues (Anderson et al., 2003; Mulvey et al., 2001), we need to think that genes encoding proteins involved in E. coli invasivity, and involved in increasing the pathogens' fitness and adaptability, either belong to the same pathogenicity islands of those mostly investigated regarding the above-mentioned relationship (Brzuszkiewicz et al., 2006), or are susceptible to the same mutational event suggested for the latter (i.e. fimA, papC, $h l y A, c n f 1$, iss, iutA etc.). If the authors took this ascertained correlation into consideration, their results could be viewed with a more critical eye and with doubt on the real significance of their findings. In my opinion, finding E. coli in urinary polymicrobial samples as the most pathogenic isolates and simultaneously as the isolates most refractory to fluoroquinolone, and finding ciprofloxacin-resistant E. coli more frequently in polymicrobial samples than in routine clinical isolates, depends on the kind of analysis carried out. In fact, the analysis by Croxall et al. (2011) only considered elderly, and therefore compromised, subjects, and did not separate catheterized from the noncatheterized patients. In our aforementioned study (Saverino et al., 2011), we also obtained, among polymicrobial UTIs, results in contrast with the literature, i.e. other than an enhanced pathogenic capability of quinolone/fluoroquinolone-susceptible E. coli, with respect to the resistant strains, a capability to suppress interleukin-8 urothelial production, a significant correlation between quinolone/ fluoroquinolone E. coli susceptibility and mixed cultures. However, we expected this discrepancy and better still we highlighted 
the paradox emerging from the latter correlation and from the

contemporaneous connection between quinolone/fluoroquinolone-resistant E. coli and compromised hosts, 'known to be frequently affected by polymicrobial UTIs'. In our work, in which urine from catheters were excluded, we tried to explain this paradox bearing in mind that pathogenesis underlying polymicrobial UTIs in a compromised host could be completely different from that in a noncompromised host, in whom mixed urinary infections would seem to be due to the pathogenicity of certain bacteria.

Lastly, as already mentioned above, in their work the authors listed the antimicrobial susceptibility phenotype of the isolates. Our interpretation of Croxall and colleagues study method is strengthened by data that they obtained, even if not statistically significant, concerning similar results for quinolone/fluoroquinolone and trimethoprim resistance, and only in E. coli isolates. The underlying mechanisms of resistance are known to be mostly different for each antibiotic and, to a lesser extent, also for each bacterial species. Particularly, it is well known that among several mechanisms by which bacteria can develop resistance to trimethoprim, clinically the most important of these is the plasmidencoded production of additional dihydrofolate reductases, and such resistance is found in both Gram-positive and Gram-negative species (Thomson, 1993). Given the prevalence of polymicrobial UTIs in the compromised host, a greater resistance of most isolate species to most of the antibacterial drugs would have been expected in mixed than in monomicrobial UTIs.

\section{Gabriella Piatti}

Section of Microbiology, Department of Surgical and Diagnostic Sciences, University of Genoa, Genoa, Italy

\section{Correspondence: Gabriella Piatti (piatti@unige.it)}

Anderson, G. G., Palermo, J. J., Schilling, J. D., Roth, R., Heuser, J. \& Hultgren, S. J. (2003). Intracellular bacterial biofilm-like pods in urinary tract infections. Science 301, 105-107.

Brzuszkiewicz, E., Brüggemann, $H_{\text {., }}$ Liesegang, H., Emmerth, M., Olschläger, T., Nagy, G., Albermann, K., Wagner, C., Buchrieser, C. \& other authors (2006). How to become a uropathogen: comparative genomic analysis of extraintestinal pathogenic Escherichia coli strains. Proc Natl Acad Sci U S A 103, 12879-12884.

Croxall, G., Weston, V., Joseph, S., Manning, G., Cheetham, P. \& McNally, A. (2011). Increased human pathogenic potential of Escherichia coli from polymicrobial urinary tract infections in comparison to isolates from monomicrobial culture samples. J Med Microbiol 60, 102-109.

Domann, E., Hong, G., Imirzalioglu, C., Turschner, S., Kühle, J., Watzel, C., Hain, T., Hossain, H. \& Chakraborty, T. (2003). Cultureindependent identification of pathogenic bacteria and polymicrobial infections in the genitourinary tract of renal transplant recipients. J Clin Microbiol 41, 5500-5510.

Foxman, B. \& Brown, P. (2003). Epidemiology of urinary tract infections: transmission and risk factors, incidence, and costs. Infect Dis Clin North Am 17, 227-241.

Houdouin, V., Bonacorsi, S., Mahjoub-Messai, F., Mariani-Kurkdjian, P., Bidet, P., Sebag, G., Loirat, C., Bourrillon, A. \& Bingen, E. (2007).
Phylogenetic groups and virulence factors of Escherichia coli strains causing pyelonephritis in children with and without urinary tract abnormalities. Clin Microbiol Infect 13, 740-742.

Johnson, J. R. (1991). Virulence factors in Escherichia coli urinary tract infection. Clin Microbiol Rev 4, 80-128.

Johnson, J. R. (2005). Virulence factors in Escherichia coli. J Clin Microbiol 43, 62216221.

Mulvey, M. A., Schilling, J. D. \& Hultgren, S. J. (2001). Establishment of a persistent Escherichia coli reservoir during the acute phase of a bladder infection. Infect Immun 69, 4572-4579.

Platell, J. L., Cobbold, R. N., Johnson, J. R. \& Trott, D. J. (2010). Clonal group distribution of fluoroquinolone-resistant Escherichia coli among humans and companion animals in Australia. J Antimicrob Chemother 65, 1936-1938.

Ronald, A. (2003). The etiology of urinary tract infection: traditional and emerging pathogens. Dis Mon 49, 71-82.

Saverino, D., Schito, A. M., Mannini, A., Penco, S., Bassi, A. M. \& Piatti, G. (2011). Quinolone/ fluoroquinolone susceptibility in Escherichia coli correlates with human polymicrobial bacteriuria and with in vitro interleukine-8 suppression. FEMS Immunol Med Microbiol 61, 84-93.

Siegman-Igra, Y., Kulka, T., Schwartz, D. \& Konforti, N. (1994). Polymicrobial and monomicrobial bacteraemic urinary tract infection. J Hosp Infect 28, 49-56.

Takahashi, A., Muratani, T., Yasuda, M., Takahashi, S., Monden, K., Ishikawa, K., Kiyota, H., Arakawa, S., Matsumoto, T. \& other authors (2009). Genetic profiles of fluoroquinoloneresistant Escherichia coli isolates obtained from patients with cystitis: phylogeny, virulence factors, PAIusp subtypes, and mutation patterns. J Clin Microbiol 47, 791-795.

Thomson, C. J. (1993). Trimethoprim and brodimoprim resistance of gram-positive and gram-negative bacteria. J Chemother 5, 458-464.

\section{Authors' reply to 'Pathogenic potential of Escherichia coli from polymicrobial urinary tract infections'}

On behalf of the authors of our recent publication on the pathogenesis of Escherichia coli from polymicrobial urinary tract infections (UTIs) (Croxall et al., 2011), we present a response to the correspondence paper by Gabriella Piatti (Piatti, 2011). We thank the author for their interest in our work, and especially welcome their comment on the importance of studying such infections. We also hereby attempt to clarify some points of confusion in the author's interpretation of the data displayed in the initial publication.

Piatti begins by stating that our analysis of antimicrobial resistance data were (probably) performed regardless of patient group. We clearly state in our manuscript that there was no difference in levels of antimicrobial resistance between polymicrobial and monomicrobial samples. As there was no statistically significant difference we chose not to present that data in detail given the amount of data we had to present on what we considered our significant findings, 